\title{
Metodología estadística para la selección de talentos \\ deportivos en estudiantes de 9 a 12 años: Un estudio de
}

caso.

\section{Statistical methodology for the selection of sports talents in students aged \\ 9 to 12 years: A case study.}

Ana Carola Flores Mancheno. ${ }^{1}$, Marco Vinicio Parra Chávez. ${ }^{2}$

Recibido: 16-05-2019 / Revisado: 19-06-2019 /Aceptado: 10-07-2019/ Publicado: 30-07-2019

\begin{abstract}
.
DOI: https://doi.org/10.33262/cienciadigital.v3i3.2.1.887

The identification of sports talents becomes extremely complex when there isn't statistical methodology that supports the selection of potential talents, especially at an early age. This research establishes a regulation of talent selection based on innate motor skills in students aged 9 to 12 who have not undergone specific preparation. The regulations were generated through the application of a control test in a sample of 1398 students from the city of Riobamba. The evaluation of students who have greater potential, allowed the identification of 370 subjects who stand out with certain conditions to be initiated in sports training, considering that due to their characteristics they can apply to more than one discipline.
\end{abstract}

Keywords: Sports initiation, control test, motor skills, statistical analysis, case study.

\section{Resumen.}

La identificación de talentos deportivos se vuelve sumamente compleja cuando no se cuenta con una metodología estadística que respalde la selección de los potenciales talentos sobre todo edades tempranas. Esta investigación establece una normativa de selección de talentos basada en las capacidades motrices innatas en estudiantes de 9 a 12 años que no han sido sometidos a una preparación específica. La normativa se generó a través de la aplicación de un test de control en una muestra de 1398 estudiantes de la ciudad de Riobamba. La evaluación a los estudiantes que poseen mayor potencial, permitió la identificación de 370 sujetos que se destacan con ciertas condiciones para

1 Escuela Superior Politécnica de Chimborazo, Facultad de Recursos Naturales. Riobamba, Ecuador. acmancheno@espoch.edu.ec

2 Escuela Superior Politécnica de Chimborazo, Facultad de Ciencias. Riobamba, Ecuador. marcov.parra@espoch.edu.ec 
ser iniciados en el entrenamiento deportivo, tomando en cuenta que por sus características pueden aplicar a más de una disciplina.

Palabras claves: Iniciación deportiva, test de control, capacidades motrices, análisis estadístico, estudio de caso.

\section{Introducción.}

La detección, selección y preparación de potenciales deportivos, constituye sin lugar a duda el método más eficaz, mediante el cual, los entrenadores logran en la actualidad rendimientos superiores en el deporte de alta competencia. Esta performance se adquiere a través de la aplicación de métodos que conllevan a desarrollar un organismo hasta niveles extremos, alcanzando éxitos, cuya representatividad se enmarca en contextos nacionales y mundiales.

Todo esto es posible siempre y cuando los sujetos que estén sometidos a un régimen de entrenamiento, sean aquellos capaces de asimilar las intensas cargas y volúmenes de trabajo, tanto en el aspecto físico como en el psicológico, ya que, la aplicación de las diversas formas de preparación deportiva, no desarrollan a los sujetos hasta el alto rendimiento si estos no tienen las características innatas necesarias para ser considerados talentos. El contar con los sujetos con un potencial deportivo superior no asegura la obtención de resultados, pero si un rendimiento adecuado.

La Federación Deportiva de Chimborazo (F.D.CH), contando con el apoyo de la Dirección Provincial de Educación Hispana de Chimborazo y la Supervisión de Educación en el área de Cultura Física, realizó la aplicación de un test de control para la observación de las capacidades físicas y antropométricas en la población no deportiva, en los estudiantes de Sexto y Séptimo años de EGB de la ciudad de Riobamba.

Por medio del análisis estadístico de los resultados obtenidos en el test, se pretende evaluar el potencial físico de los estudiantes, permitiendo diagnosticar sus capacidades motrices, determinar su nivel de eficiencia física para ayudar en la toma de decisiones de la selección de los posibles deportistas de alto rendimiento.

Por la experiencia de los estudios realizados en Cuba, Nicaragua, Brasil y México, el Dr. Hermenegildo Pila Hernández, experto en la Selección de talentos para el deporte, con 27 
años de experiencia en Cuba, sugiriere que cada país y región establezcan sus propias normas de evaluación, pues existen diferencias que se han podido constatar entre países en cuanto a las marcas de evaluación, diferencias que están dadas por muchos factores, entre ellos, las características geográfica, medio ambientales y el desarrollo socioeconómico, entre otros.

\section{Talento deportivo}

Para Gabler y Ruoff (1979; en Baur, 1993, p. 6), "un talento deportivo es aquel que, en un determinado estado de la evolución, se caracteriza por determinadas condiciones y presupuestos físicos y psíquicos, los cuales, con mucha probabilidad, le llevarán, en un momento sucesivo, a alcanzar prestaciones de alto nivel en un determinado tipo de deporte". (Baur, 1993). Así también Zatsiorski (1989), afirma que "el talento deportivo se caracteriza por determinada combinación de las capacidades motoras y psicológicas, así como de las aptitudes anatomofisiológicas que crean, en conjunto, la posibilidad potencial para el logro de altos resultados deportivos en un deporte concreto".

\section{Estrategias de selección}

Pila (2003), considera que en la actualidad existen tres formas empíricas que los entrenadores y profesores de educación física aplican para seleccionar talentos:

1. La que se produce cuando los entrenadores deportivos asisten a las competencias que se desarrollan en el ámbito escolar, en ellas observa los rendimientos o la participación destacada de los competidores y eligen de esta manera, los elementos que integraran la selección para sus grupos de trabajo.

1. Esta forma tiene en cuenta la opinión del Profesor de Educación Física, cuando el entrenador de un deporte se le acerca a preguntarle si posee algún alumno que reúna ciertas y determinadas características requeridas para su deporte en cuestión y el Profesor de Educación Física que conoce el desarrollo en capacidades y habilidades de la matrícula que atiende, le señala particularmente aquellos que se acercan a los requerimientos planteados. 
2. Se trata de la más empírica de las formas, es aquella en la que el Entrenador Deportivo, simplemente en cualquier lugar, en la calle, un parque o una actividad social, observa en un niño o adolescente alguna característica somatotipológica, disposición o aptitud que le hace determinar un posible desarrollo en su deporte.

Estas son las tres formas que actualmente se aplican en cualquier latitud, todas empíricas y carentes de rigor en valoraciones con carácter científico de evaluación, que permita una consideración en proyecciones y perspectivas sobre bases sólidas para establecer un diagnóstico adecuado

Aparece una nueva forma, la cuarta, que se sustenta sobre bases más científicas (aplicación de las técnicas estadísticas) y parte de un principio masivo en su aplicación, esta forma, las pruebas de valoración física o de rendimiento motor que se aplica por los Profesores de Educación Física en las escuelas, permite a través de sus normas de evaluación, establecer un sistema de clasificación de las potencialidades motrices y somatotipológicas para una adecuada iniciación en la práctica de los deportes.

Indistintamente de la metodología que evalúe los talentos, se debe tomar en cuenta que el resultado competitivo puede estar dado por múltiples factores, entre los que conjugan los factores subjetivos y objetivos, no se debe tener a éste como único factor de selección, en este sentido, cuando resulta imposible poner en marcha un sistema de selección de multi enfoque donde se evalúen capacidades, habilidades, etc., se deberá hacer un análisis estructurado y sistémico del comportamiento competitivo en un período prolongado de tiempo, sobre la base de datos tomados por los propios seleccionadores. (Leyva Infante, 2003)

\section{Preparación deportiva}

Generalmente se da en dos etapas principales: 1) La general, que trabaja cualidades físicas, como la fuerza, la resistencia, la velocidad, la flexibilidad, los reflejos que pretende mantener una alta capacidad de rendimiento físico y psíquico; además de proporciona un desarrollo equilibrado de todos los grupos musculares, de todas las funciones orgánicas y sistemas 
energéticos; 2) La específica se refiere al desarrollo de las cualidades físicas, grupos musculares, sistemas energéticos, funciones orgánicas, movimientos especiales y adecuaciones del trabajo a las necesidades particulares de una determinada actividad deportiva. Sin una buena preparación física general, es casi imposible realizar una preparación física específica eficaz, debido a que ésta se basa en el nivel físico adquirido previamente.

La preparación deportiva se compone por el trabajo de un equipo interdisciplinario donde, los entrenadores y metodólogos encaminan su esfuerzo hacia un mismo fin, mismo que pretende alcanzar los máximos rendimientos deportivos. (Sánchez Rodríguez \& Gutiérrez Gómez, 2014)

\section{Metodología}

\section{Área de estudio}

La presente investigación se desarrolló en el Departamento de Estadística de la Unidad de Gestión Técnica Metodológica de la Federación Deportiva de Chimborazo. La población en este estudio estuvo compuesta por los 9415 estudiantes de sexto y séptimo año de Educación Básica de la ciudad de Riobamba, los mismos que están en las edades comprendidas entre los 9 y 12 años de edad, de ellos 4601 son de género masculino y 4814 femenino.

\section{Recolección de datos}

Se desarrolló un estudio de campo, donde se contó con la participación de 26 escuelas (fiscales y particulares); la técnica utilizada para la recopilación de la información fue un test de control que contó con tres apartados: a) Datos informativos, b) Medidas antropométricas, c) Pruebas físicas. Este test fue aplicado a 1398 estudiantes, es decir, el $14,85 \%$ de la población. Las variables medidas fueron cualitativas-cuantitativas y los datos son de corte transversal.

Para la aplicación del test se trabajó diariamente con un número de 2 a 4 escuelas, las pruebas a los estudiantes se aplicaron en una sola sesión de trabajo, en grupos de 10 a 20 estudiantes para asegurar la calidad y confiabilidad de las mismas. Para ello, los estudiantes se 
presentaron con vestuario y calzado deportivo (pantalón corto, camiseta y zapatos tenis) a fin de no obstaculizar la ejecución técnica del movimiento. Cabe mencionar que, todos estuvieron aptos físicamente y no contaban con algún padecimiento o enfermedad que los eximiera de la práctica de ejercicios físicos.

El orden de realización fue el siguiente: Recepción de estatura de pie (talla), estatura sentado (E.S), peso, envergadura (ENV.) y perímetro abdominal (P.ABD.). Calentamiento, velocidad (V 30m.), fuerza en extremidades superiores (lagartijas, F.B.), fuerza en abdomen (abdominales, F.A.), fuerza en extremidades inferiores (salto de longitud sin carrera de impulso, SLSCI) y resistencia (R 1000m).

La aplicación del test se realizó a partir de las 8 am, las pruebas que lo conforman se distribuyeron en el Estadio Olímpico de Riobamba a manera de estaciones, los entrenadores que las realizaron contaron con una capacitación previa de la normativa de aplicación y los resultados de cada estudiante se anotaron en una ficha con la información de la escuela y año al cual correspondían.

\section{Análisis de datos}

Se inicia el análisis a través de la descripción del número de hombres y mujeres presentes en la muestra, así como de los resultados obtenidos en las pruebas antropométricas y físicas. Posteriormente, se elabora la normativa para la selección de talentos a través de los percentiles obtenidos por género y edad de los estudiantes. En el caso de la estatura la normativa, fue elaborada utilizando el percentil 97 de la presente investigación; en las tablas restantes, aparecen los valores de los percentiles 10 al 90 que son utilizados para evaluar los resultados en cada capacidad motriz o prueba medida.

Para obtener la evaluación general por puntos, se suma el percentil alcanzado por cada estudiante de acuerdo al resultado obtenido en las pruebas físicas y se los clasifica de acuerdo a la tabla que se muestra a continuación: 
Tabla 1. Tabla de evaluación general por puntos

\begin{tabular}{lc}
\hline \multicolumn{1}{c}{ EVALUACIÓN } & CRITERIO \\
\hline \hline Muy Bueno & 330 o más \\
\hline Bueno & 240 a 320 \\
\hline Deficiente & 150 a 230 \\
\hline Regular & 60 a 140 \\
\hline Malo & 50 o menos \\
\hline
\end{tabular}

Fuente: Pila Hernández H., Selección de talentos para el deporte.

Elaborado por: Los autores

\section{Resultados}

La Figura 2 presenta la distribución muestral de esta investigación, la misma que estuvo compuesta del $55 \%$ de estudiantes de género femenino y un $45 \%$ de género masculino; las edades fluctúan entre 9 y 12 años, siendo 10 años la edad que se presenta con mayor frecuencia. Los estudiantes de 9 a 11 años representan aproximadamente el $98 \%$ de la muestra.

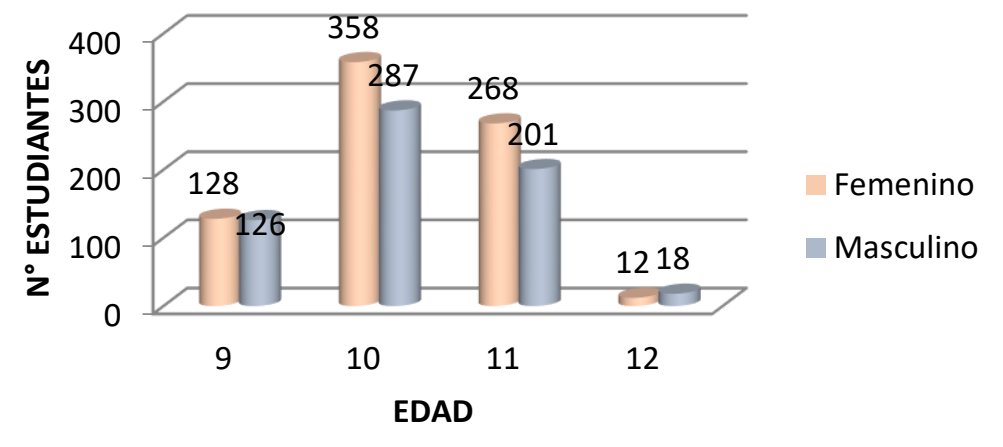

Figura 1. Número de estudiantes por edad y género Fuente: Trabajo de campo

Elaborado por: Los autores

Los resultados que se presentan en las tablas 2 y 3 demuestran una similitud en las características antropométricas de los estudiantes de ambos géneros: peso aproximado $34 \mathrm{~kg}$ y talla de $137 \mathrm{~cm}$ en promedio; sin embargo, en las pruebas físicas, los hombres tienen ciertos rasgos de supremacía al ser más veloces (5,18 vs 5,51 segundos en la prueba V 30m), más fuertes (12,77 vs 9,95 repeticiones en la prueba F.B; 19,37 vs 16,20 repeticiones en la prueba F.A. y 153,33 vs $142,11 \mathrm{~cm}$ en la prueba SLSCI) y con mayor resistencia (335,33 vs 389,83 segundos en la prueba R 1000m) 
Tabla 2. Estadísticas descriptivas del test de control aplicado a los estudiantes de género femenino

\begin{tabular}{|c|c|c|c|c|c|c|c|c|c|c|c|}
\hline & EDAD & PESO & TALLA & P. ABD. & ENV. & E.S. & V $30 \mathrm{~m}$. & F.B. & F. A. & R $1000 \mathrm{~m}$ & SLSCI \\
\hline Media & 10,214 & 34,489 & 137,438 & 63,338 & 136,356 & 71,246 & 5,514 & 9,953 & 16,205 & 389,829 & 142,109 \\
\hline Error típico & 0,026 & 0,272 & 0,261 & 0,279 & 0,315 & 0,162 & 0,022 & 0,193 & 0,195 & 2,536 & 0,739 \\
\hline Mediana & 10 & 33,3 & 137 & 62 & 136 & 71 & 5,48 & 11 & 16 & 388 & 142 \\
\hline Moda & 10 & 31,2 & 137 & 60 & 136 & 69 & 4,92 & 14 & 15 & 371 & 145 \\
\hline Desv. Estándar & 0,731 & 7,532 & 7,237 & 7,710 & 8,711 & 4,497 & 0,618 & 5,336 & 5,407 & 69,626 & 20,461 \\
\hline Coef. asimetría & $-0,115$ & 0,935 & 0,215 & 0,814 & 0,098 & $-0,356$ & 0,611 & $-0,051$ & $-0,069$ & 0,218 & $-0,042$ \\
\hline Rango & 3 & 44,65 & 46 & 48 & 59 & 50 & 3,9 & 28 & 45 & 451 & 126 \\
\hline Mínimo & 9 & 20 & 115 & 47 & 107 & 47 & 4,03 & 0 & 0 & 229 & 74 \\
\hline Máximo & 12 & 64,65 & 161 & 95 & 166 & 97 & 7,93 & 28 & 45 & 680 & 200 \\
\hline Cuenta & 766 & 766 & 766 & 766 & 766 & 766 & 766 & 766 & 766 & 754 & 766 \\
\hline $\mathrm{CV}$ & & $21,8 \%$ & $5,3 \%$ & $12,2 \%$ & $6,4 \%$ & $6,3 \%$ & $11,2 \%$ & $53,6 \%$ & $33,4 \%$ & $17,9 \%$ & $14,4 \%$ \\
\hline
\end{tabular}

Tabla 3. Estadísticas descriptivas del test de control aplicado a los estudiantes de género masculino

\begin{tabular}{|c|c|c|c|c|c|c|c|c|c|c|c|}
\hline & EDAD & PESO & TALLA & P. ABD. & ENV. & E.S. & V $30 \mathrm{~m}$. & F.B. & F. A. & R $1000 \mathrm{~m}$ & SLSCI \\
\hline Media & 10,176 & 33,407 & 136,824 & 64,578 & 135,445 & 70,764 & 5,180 & 12,778 & 19,369 & 335,333 & 153,334 \\
\hline Error típico & 0,031 & 0,288 & 0,267 & 0,318 & 0,325 & 0,157 & 0,022 & 0,183 & 0,203 & 2,667 & 0,754 \\
\hline Mediana & 10 & 31,825 & 137 & 63 & 135 & 71 & 5,12 & 13 & 20 & 325 & 154 \\
\hline Moda & 10 & 30,75 & 137 & 63 & 137 & 71 & 5,09 & 14 & 20 & 325 & 150 \\
\hline Desv. estándar & 0,776 & 7,244 & 6,704 & 7,990 & 8,165 & 3,956 & 0,554 & 4,601 & 5,115 & 66,824 & 18,965 \\
\hline Coef. asimetría & 0,053 & 1,509 & 0,161 & 1,220 & 0,268 & $-0,568$ & 1,008 & $-0,099$ & 0,260 & 0,826 & $-0,269$ \\
\hline Rango & 3 & 55,9 & 47,6 & 53 & 51 & 36 & 5,09 & 26 & 43 & 365 & 111 \\
\hline Mínimo & 9 & 21,1 & 114 & 50 & 114 & 52 & 3,72 & 0 & 2 & 225 & 97 \\
\hline Máximo & 12 & 77 & 161,6 & 103 & 165 & 88 & 8,81 & 26 & 45 & 590 & 208 \\
\hline Cuenta & 632 & 632 & 632 & 632 & 632 & 632 & 632 & 632 & 632 & 628 & 632 \\
\hline $\mathrm{CV}$ & & $21,7 \%$ & $4,9 \%$ & $12,4 \%$ & $6,0 \%$ & $5,6 \%$ & $10,7 \%$ & $36,0 \%$ & $26,4 \%$ & $19,9 \%$ & $12,4 \%$ \\
\hline
\end{tabular}

Fuente: Trabajo de campo

Elaborado por: Los autores

Indicadores deportivos para los estudiantes de sexto y séptimo años de EGB de la ciudad de Riobamba

Los indicadores deportivos presentes en las tablas 4, 5 y 6, serán el punto de referencia para evaluar el desarrollo de las capacidades motrices a los estudiantes de sexto y séptimo años de EGB de géneros femenino y masculino, en edades comprendidas entre los 9 y 12 años. Por lo tanto, estos indicadores serán considerados como Normativa de Selección de Talentos.

Tabla 4. Normativa de selección de talentos en estatura

\begin{tabular}{ccc}
\hline \multicolumn{3}{c}{ TALLA $(\mathbf{c m})$} \\
\hline EDAD & MASCULINO & FEMENINO \\
$\mathbf{9}$ & 144,6 & 147,6 \\
$\mathbf{1 0}$ & 148,4 & 148,7 \\
$\mathbf{1 1}$ & 150,5 & 155,6 \\
$\mathbf{1 2}$ & 157,2 & 156,1 \\
\hline
\end{tabular}

Fuente: Trabajo de campo

Elaborado por: Los autores 


\section{C) Ciencia

ISSN: 2602-8085

www.cienciadigital.org

Vol. 3, N³.2.1, p. 323-337, julio, 2019

Tabla 5. Normativa de selección de talentos en capacidades motrices, estudiantes de género femenino

\begin{tabular}{|c|c|c|c|c|c|c|c|c|c|}
\hline \multicolumn{10}{|c|}{ PERCENTILES } \\
\hline EDAD & 10 & 20 & 30 & 40 & 50 & 60 & 70 & 80 & 90 \\
\hline 9 & 6,35 & 6,05 & 5,82 & 5,64 & 5,57 & 5,41 & 5,26 & 5,07 & 4,92 \\
\hline 10 & 6,37 & 6,05 & 5,81 & 5,67 & 5,51 & 5,37 & 5,18 & 4,99 & 4,84 \\
\hline 11 & 6,24 & 5,92 & 5,7 & 5,52 & 5,35 & 5,22 & 5,09 & 4,94 & 4,73 \\
\hline 12 & 6,24 & 5.92 & 5,7 & 5,52 & 5,35 & 5,22 & 5,09 & 4.94 & 4,73 \\
\hline
\end{tabular}

욘

\begin{tabular}{|c|c|c|c|c|c|c|c|c|c|}
\hline \multicolumn{10}{|c|}{ PERCENTILES } \\
\hline EDAD & 10 & 20 & 30 & 40 & 50 & 60 & 70 & 80 & 90 \\
\hline 9 & 3 & 6 & 10 & 11 & 12 & 13 & 14 & 14 & 16 \\
10 & 3 & 5 & 8 & 10 & 11 & 12 & 13 & 14 & 15 \\
11 & 2 & 3 & 5 & 7 & 9 & 11 & 12 & 14 & 15 \\
12 & 2 & 3 & 5 & 7 & 9 & 11 & 12 & 14 & 15 \\
\hline
\end{tabular}

$\mathbb{4}$

\begin{tabular}{|c|c|c|c|c|c|c|c|c|c|}
\hline \multicolumn{10}{|c|}{ PERCENTILES } \\
\hline EDAD & 10 & 20 & 30 & 40 & 50 & 60 & 70 & 80 & 90 \\
\hline $\mathbf{9}$ & 10 & 12 & 14 & 15 & 16 & 17 & 19 & 20 & 23 \\
\hline 10 & 10 & 13 & 14 & 15 & 16 & 17 & 19 & 20 & 21 \\
\hline 11 & 10 & 13 & 14 & 15 & 16 & 17 & 18 & 20 & 23 \\
\hline 12 & 10 & 13 & 14 & 15 & 16 & 17 & 18 & 20 & 23 \\
\hline
\end{tabular}

\begin{tabular}{|c|c|c|c|c|c|c|c|c|c|}
\hline \multicolumn{10}{|c|}{ PERCENTILES } \\
\hline EOAD & 10 & 20 & 30 & 40 & 50 & 60 & 70 & 80 & 90 \\
\hline 9 & 7,58 & 7,36 & 7,17 & 6,57 & 6,36 & 6,23 & 6,05 & 5,4 & 5,19 \\
\hline 10 & 8,05 & 7,33 & 7,15 & 6,55 & 6,32 & 6,1 & 5,49 & 5,29 & 5,04 \\
\hline 11 & 8,03 & 7,26 & 7,01 & 6,42 & 6,15 & 5,53 & 5,33 & 5,14 & 4,51 \\
\hline 12 & 8,03 & 7,26 & 7,01 & 6,42 & 6,15 & 5,53 & 5,33 & 5,14 & 4,51 \\
\hline
\end{tabular}

\begin{tabular}{|c|c|c|c|c|c|c|c|c|c|}
\hline \multicolumn{10}{|c|}{ PERCENTILES } \\
\hline EDAD & 10 & 20 & 30 & 40 & 50 & 60 & $\overline{70}$ & 80 & 90 \\
\hline 9 & 113,4 & 121,4 & 129 & 133,8 & 137 & 140,4 & 145,9 & 151 & 158,3 \\
\hline 10 & 115 & 124 & 130 & 136 & 142 & 147 & 153 & 160 & 170,3 \\
\hline 11 & 119 & 128 & 135,1 & 141 & 145 & 148,2 & 155 & 161 & 169 \\
\hline 12 & 120,1 & 123 & 131,8 & 133 & 139,3 & 145,4 & 148 & 159,6 & 166,2 \\
\hline
\end{tabular}

Fuente: Trabajo de campo

Elaborado por: Los autores

Tabla 6. Normativa de selección de talentos en capacidades motrices, estudiantes de género masculino

\begin{tabular}{|c|c|c|c|c|c|c|c|c|c|}
\hline \multicolumn{10}{|c|}{ PERCENTILES } \\
\hline EDAD & 10 & 20 & 30 & 40 & 50 & 60 & 70 & 80 & 90 \\
\hline 9 & 5,9 & 5,6 & 5,43 & 5,32 & 5,2 & 5,09 & 4.95 & $\frac{.53}{483}$ & 469 \\
\hline 10 & 5,93 & 5,56 & 5,41 & 5,24 & 5,13 & 5,07 & 4,95 & 4,83 & 4,62 \\
\hline 11 & 5,76 & 5,52 & 5,37 & 5,26 & 5,07 & 4,92 & 4,77 & 4,63 & 4.51 \\
\hline 12 & 5.21 & 5,07 & 5,02 & 4.99 & 4.95 & 4.81 & 4.75 & 4.61 & 4.36 \\
\hline
\end{tabular}

$\mathbb{4 0} 4$\begin{tabular}{|c|c|c|c|c|c|c|c|c|c|}
\hline \multicolumn{10}{|c|}{ PERCENTILS } \\
\hline EDAD & 10 & 20 & 30 & 40 & 50 & 60 & 70 & 80 & 90 \\
\hline 9 & 8 & 9 & 11 & 12 & 13 & 14 & 15 & 16 & 20 \\
\hline 10 & 8 & 10 & 11 & 12 & 13 & 14 & 15 & 16 & 17 \\
11 & 5 & 10 & 11 & 12 & 13 & 14 & 15 & 16 & 18 \\
\hline 12 & 11 & 12 & 13 & 14 & 15 & 17 & 19 & 20 & 21 \\
\hline
\end{tabular}

\begin{tabular}{|c|c|c|c|c|c|c|c|c|c|}
\hline \multicolumn{10}{|c|}{ PERCENTILES } \\
\hline EDAD & 10 & 20 & 30 & 40 & 50 & 60 & 70 & 80 & 90 \\
\hline 9 & 14 & 16 & 17 & 18 & 19 & 20 & 21 & 22 & 24 \\
10 & 14 & 15 & 17 & 18 & 19 & 20 & 22 & 23 & 25 \\
11 & 14 & 15 & 17 & 19 & 20 & 21 & 22 & 24 & 26 \\
12 & 13 & 17 & 19 & 21 & 22 & 23 & 24 & 25 & 26 \\
\hline
\end{tabular}
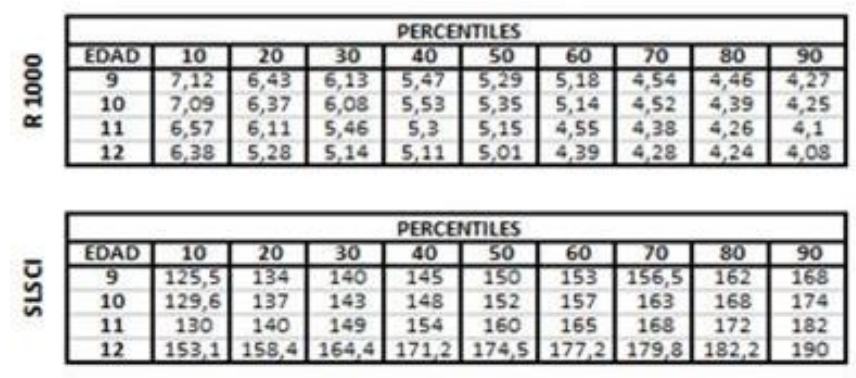

Fuente: Trabajo de campo

Elaborado por: Los autores 
Una vez aplicada la normativa de evaluación de talentos en capacidades motrices (tabla 5 y 6), se resumen en la tabla 7 el número de deportista de acuerdo a la evaluación por puntos. Los estudiantes que obtuvieron la evaluación de Muy Bueno (239), representan el 17\% de la muestra, son considerados potencialmente talentos en capacidades motrices, los mismos que deben ser convocados previo el análisis de los percentiles y someterse a un examen antropométrico, el cual determinará su potencial físico para el ingreso a las selecciones provinciales como futuros talentos deportivos, los que garantizarán el proceso de preparación deportiva.

Los estudiantes que obtuvieron la evaluación de Bueno (457), representan el 33\% de la muestra, estos serán considerados de la misma manera con el análisis respectivo sobre la capacidad física determinante en el deporte a elección, es decir, si su cualidad física es entrenable, pero su crecimiento corporal se presenta altamente desarrollado, se considerará en función de la importancia que represente para cualquiera de los grupos por deporte.

Tabla 7. Número de deportistas distribuidos por la evaluación general por puntos

\begin{tabular}{|c|c|c|c|c|c|c|}
\hline \multirow{2}{*}{ EVALUACIÓN } & \multicolumn{2}{|c|}{ FEMENINO } & \multicolumn{2}{|c|}{ MASCULINO } & \multirow{2}{*}{$\begin{array}{c}\text { FRECUENCIA } \\
\text { TOTAL }\end{array}$} & \multirow{2}{*}{$\begin{array}{c}\text { PORCENTAJE } \\
\text { TOTAL }\end{array}$} \\
\hline & Frecuencia & Porcentaje & Frecuencia & Porcentaje & & \\
\hline MUY BUENO & 146 & $19 \%$ & 93 & $15 \%$ & 239 & $17 \%$ \\
\hline BUENO & 227 & $30 \%$ & 230 & $36 \%$ & 457 & $33 \%$ \\
\hline REGULAR & 236 & $31 \%$ & 193 & $31 \%$ & 429 & $31 \%$ \\
\hline DEFICIENTE & 135 & $18 \%$ & 91 & $14 \%$ & 226 & $16 \%$ \\
\hline MALO & 22 & $3 \%$ & 25 & $4 \%$ & 47 & $3 \%$ \\
\hline Total & 766 & $100 \%$ & 632 & $100 \%$ & 1398 & $100 \%$ \\
\hline
\end{tabular}

Fuente: Trabajo de campo

Elaborado por: Los autores

Tomando como instrumento base las tablas 8 a 11, en las cuales se encuentra la Clasificación de las pruebas para las evaluaciones especiales por deportes, se procedió a la distribución de los estudiantes a las diferentes disciplinas deportivas, considerando como talentos, a aquellos sujetos que cumplan con el percentil 90 en las capacidades motrices y 97 en estatura; cabe anotar, que un estudiante en función de sus capacidades motrices, puede clasificar en una o varias disciplinas. 
Tabla 8. Clasificación de las pruebas para deportes de resistencia y fuerza rápida

\begin{tabular}{|c|c|c|}
\hline DEPORTE & PRUEBA & $\begin{array}{l}\text { CAPACIDAD MOTRIZ QUE SE } \\
\text { REQUIERE DE LAS PRUEBAS }\end{array}$ \\
\hline \multirow{3}{*}{ Atletismo } & V 30 & \\
\hline & R 1000 & Depende el evento \\
\hline & $\mathrm{SLSCI}$ & \\
\hline \multirow{2}{*}{ Ciclismo } & R 1000 & Resistencia (aeróhica) \\
\hline & SLSCI & 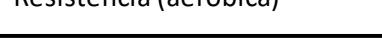 \\
\hline \multirow[t]{2}{*}{ Levantamiento de Pesas } & $\mathrm{SLSCl}$ & Fuerza rápida \\
\hline & TALLA & \\
\hline \multirow[t]{3}{*}{ Natación } & FB & Resistencia a la fuerza \\
\hline & $\mathrm{FA}$ & \\
\hline & V 30 & Resistencia (aeróbica) \\
\hline \multirow[t]{2}{*}{ Patinaje } & R 1000 & Velocidad (anaeróbica) \\
\hline & SLSCI & Fuerza rápida \\
\hline
\end{tabular}

Fuente: Hermenegildo Pila

Elaborado por: Los autores

Tabla 9. Clasificación de las pruebas para deportes con pelota

\begin{tabular}{lll}
\hline \hline \multicolumn{1}{c}{ DEPORTE } & PRUEBA & \multicolumn{1}{c}{$\begin{array}{c}\text { CAPACIDAD MOTRIZ QUE SE } \\
\text { REQUIERE DE LAS PRUEBAS }\end{array}$} \\
\hline \hline Baloncesto & TALLA & Fuerza explosiva \\
\hline \multirow{3}{*}{ Futbol } & SLSCI & Resistencia (aeróbica) \\
& R 1000 & Velocidad (anaeróbica) \\
\hline \multirow{2}{*}{ Tenis de Mesa 30} & TALLA & \\
& V 30 & Velocidad (anaeróbica) \\
\hline \multirow{2}{*}{ Voleibol de Sala o Playa } & FB & Resistencia a la fuerza \\
\hline
\end{tabular}

Fuente: Hermenegildo Pila

Elaborado por: Los autores

Tabla 10. Clasificación de las pruebas para deportes de combate

\begin{tabular}{lll}
\hline \hline \multicolumn{1}{c}{ DEPORTE } & PRUEBA & $\begin{array}{c}\text { CAPACIDAD MOTRIZ QUE SE } \\
\text { REQUIERE DE LAS PRUEBAS }\end{array}$ \\
\hline \hline Boxeo & V 30 & Velocidad (anaeróbica) \\
\hline \multirow{2}{*}{ Judo } & FB & Velocidad (anaeróbica) \\
& FA & Resistencia a la fuerza \\
\hline \multirow{2}{*}{ Karate Do } & V 30 & \\
\hline \multirow{2}{*}{ Lucha Olímpica } & TALLA & Velocidad (anaeróbica) \\
& FB & Velocidad (anaeróbica) \\
\hline \multirow{2}{*}{ Tae Kwon Do } & FA & Resistencia a la fuerza \\
\hline & V 30 & \\
\hline
\end{tabular}

Fuente: Hermenegildo Pila

Elaborado por: Los autores 
Tabla 11. Clasificación de las pruebas para deportes de apreciación y arte competitivo

\begin{tabular}{lll}
\hline \hline \multicolumn{1}{c}{ DEPORTE } & PRUEBA & $\begin{array}{c}\text { CAPACIDAD MOTRIZ QUE SE } \\
\text { REQUIERE DE LAS PRUEBAS }\end{array}$ \\
\hline \hline \multirow{2}{*}{ Gimnasia Artística } & V 30 & Velocidad (anaeróbica) \\
& FB & Resistencia a la fuerza \\
& FA & \\
\hline \multirow{2}{*}{ Gimnasia Rítmica } & FB & Resistencia a la fuerza \\
\hline \multirow{2}{*}{ Tiro con Arco } & FA & \\
& FB & \\
\hline \multirow{2}{*}{ Tiro Deportivo } & FA & Resistencia a la fuerza \\
\hline
\end{tabular}

Fuente: Hermenegildo Pila

Elaborado por: Los autores

En la tabla 12, se detalla el número de estudiantes fueron distribuidos a las diferentes disciplinas deportivas tomando en cuenta el desarrollo de sus capacidades motrices.

Tabla 12. Número de estudiantes distribuidos en las disciplinas deportivas

\begin{tabular}{|c|c|c|c|c|c|c|c|}
\hline & \multirow[b]{2}{*}{ TALLA } & & & & & & \\
\hline & & V30 & FB & FA & R1000 & SLSCI & FRECUENCIA \\
\hline ATLETISMO & & $X$ & & & $X$ & $X$ & $335^{*}$ \\
\hline PATINAJE & & $x$ & & & $x$ & $x$ & 15 \\
\hline CICLISMO & & & & & $x$ & $x$ & 33 \\
\hline PESAS & & $x$ & $x$ & & & $x$ & 15 \\
\hline $\begin{array}{l}\text { TIRO CON ARCO } \\
\text { NATACIÓN }\end{array}$ & $x$ & & $x$ & $x$ & & & $0 * *$ \\
\hline $\begin{array}{l}\text { TIRO DEPORTIVO } \\
\text { GIMN. RÍTMICA }\end{array}$ & & & $x$ & $x$ & & & 62 \\
\hline LUCHA & & & & & & & \\
\hline $\begin{array}{l}\text { TENIS DE MESA } \\
\text { GIMN. ARTÍSTICA }\end{array}$ & & $x$ & $x$ & $x$ & & & 15 \\
\hline $\begin{array}{l}\text { TAE KWON DO } \\
\text { KARATE DO }\end{array}$ & $x$ & $x$ & & & & & 6 \\
\hline $\begin{array}{l}\text { FUTBOL } \\
\text { (ARQUERO) }\end{array}$ & $\mathrm{X}$ & $\mathrm{X}$ & & & $x$ & & $43(2) * * *$ \\
\hline $\begin{array}{l}\text { BALONCESTO } \\
\text { VOLEIBOL }\end{array}$ & $x$ & & & & & $x$ & 8 \\
\hline JUDO & & $x$ & & $x$ & & & 32 \\
\hline BOXEO & & $x$ & $x$ & & & & 34 \\
\hline
\end{tabular}

X (opcional)

Fuente: Trabajo de campo

Elaborado por: Los autores 
* En la disciplina de Atletismo, se registran 335 sujetos que cumplen con al menos uno de los requerimientos para este deporte, es decir, cumplen con el percentil 90 en alguna de estas capacidades motrices V30, R1000 o SLSCI.

** Los requerimientos de las disciplinas Tiro con Arco y Natación son cumplir con el percentil 97 en TALLA y con el percentil 90 en FB y FA, al no haber sujetos que cumplan con estas tres capacidades motrices, la frecuencia para estas disciplinas es 0.

*** En la disciplina de Fútbol, se registran 43 sujetos que cumplen con el percentil 90 en las capacidades motrices V30 y R1000, asimismo hay dos sujetos que adicional a estas capacidades motrices cumplen con el percentil 97 en estatura, los cuales pueden ser considerados para arqueros.

\section{Conclusiones.}

- El análisis estadístico realizado, ayudó a la detección de sujetos considerados como potenciales talentos en capacidades motrices, a través de los resultados obtenidos por la aplicación del test de control.

- Por medio de los indicadores obtenidos a través de las técnicas estadísticas aplicadas en esta investigación, se estableció la normativa de evaluación para los estudiantes de sexto y séptimo años de EGB de la ciudad de Riobamba, en edades comprendidas entre los 9 y 12 años.

- La evaluación con técnicas estadísticas a los estudiantes que poseen mayor potencial deportivo, permitió la identificación de 370 estudiantes que cumplen con las capacidades motrices necesarias para ser iniciados en el entrenamiento deportivo de F.D.CH.

- Tomando en cuenta que por sus características pueden aplicar a más de una disciplina, se clasificaron a los estudiantes en las siguientes disciplinas: Atletismo 335, Patinaje 15, Ciclismo 33, Halterofilia 15, Lucha, Tenis de Mesa o Gimnasia Artística 15, Tae Kwon Do o Karate Do 6, Futbol 43, Baloncesto o Voleibol 8, Judo 32, Boxeo 34.

\section{Referencias bibliográficas.}

Baur, J. (1993). Ricerca e promozione del talento nello sport. Rivista di Cultura Sportiva, suplemento a SdS, 28-29, p. 4-20. 
Leyva Infante, R. (2003). La selección de talentos deportivos. Criterios para asegurar su eficacia. efdeportes. Recuperado el 12 de julio de 2016, de https://www.efdeportes.com/efd61/talento.htm

Pila Hernández, H. (2003). Selección de talentos para el deporte, 27 años de experiencia en Cuba. efdeportes. Recuperado el 13 de octubre de 2015, de http://www.efdeportes.com/efd62/talento.htm

Pila Hernández, H. (2004). Selección de talentos para el deporte, 27 años de experiencia en Cuba, metodología para evaluar las pruebas. efdeportes. Recuperado el 22 de septiembre de 2014, de https://www.efdeportes.com/efd69/talento.htm

Sánchez Rodríguez, D. \& Gutiérrez Gómez, C. (2014). Hacia una conceptualización de la preparación deportiva: elementos y roles. efdeportes. Recuperado el 23 de julio de 2016, de https://www.efdeportes.com/efd190/la-preparacion-deportiva-elementos-yroles.htm

Zatziorski, V. M. (1989). Metrología deportiva. Moscú: Planeta.

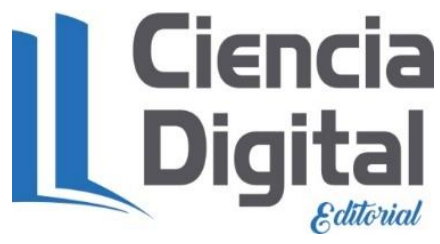




\section{PARA CITAR EL ARTÍCULO INDEXADO.}

Flores Mancheno, A., \& Parra Chávez, M. (2019). Metodología estadística para la selección de talentos deportivos en estudiantes de 9 a 12 años: Un estudio de caso. Ciencia Digital, 3(3.2.1), 323337. https://doi.org/10.33262/cienciadigital.v3i3.2.1.887

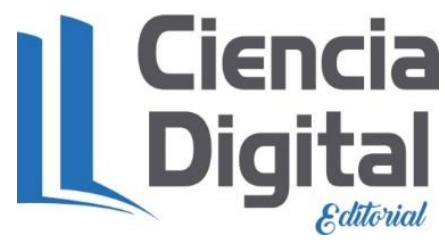

El artículo que se publica es de exclusiva responsabilidad de los autores y no necesariamente reflejan el pensamiento de la Revista Ciencia Digital.

El artículo queda en propiedad de la revista y, por tanto, su publicación parcial y/o total en otro medio tiene que ser autorizado por el director de la Revista Ciencia Digital.
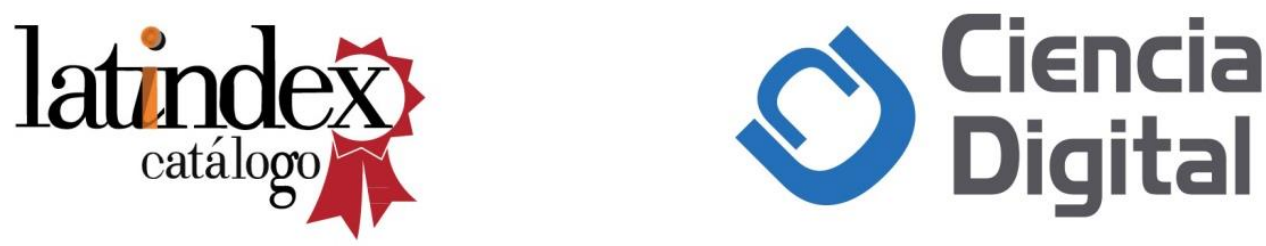